\title{
Changes in acridine orange binding and its use in the characterisation of heterochromatic regions
}

\author{
J. L. Bella, C. García de la Vega, \\ C. López-Fernández and J. Gosálvez
}

\begin{abstract}
Dpto. de Genética C-XV, Facultad de Ciencias, Universidad Autónoma de Madrid, Madrid 28049, Spain
\end{abstract}

Heterochromatic regions of the mountain grasshopper Arcyptera fusca have been stained with acridine orange (A.O.) under different conditions. Results show a certain amount of differentiation between centromeric and telomeric regions as regards their staining properties. Thus, A.O. seems able to differentiate homogeneous C-bands. In this response not only the DNA but also the fraction of proteins which remain in the chromatin may be involved.

\section{INTRODUCTION}

The employment of fluorochromes in the comparative cytological analysis of fixed chromosomes has revealed the existence of large differences within the chromatin which appeared either homogeneously or differentially stained with standard and banding techniques respectively (Caspersson et al., 1969; John et al., 1985). Thus, now it is possible to carry out detailed analyses of AT or $\mathrm{GC}$ enriched heterochromatic regions by using specific DNA binding fluorescent dyes (Schweizer, 1981). All these techniques have yielded a more dynamic view of certain regions of the chromosome usually considered as plain constitutive heterochromatin. These methods have provided several data on the response of C-positive material to specific fluorochromes in plants (Schweizer, 1976; Sato, 1980), grasshoppers (Schweizer et al., 1983; John et al., 1985) and mammals (Schnedl et al., 1981).

Acridine orange (A.O.) is a fluorescent dye which has been used in cytological studies for different purposes. A.O. binds to the DNA by intercalation between adjacent base pairs and by electrostatic interaction with phosphate groups outside the DNA (Sakoda et al., 1971; Stockert and Lisanti, 1972). The metachromasia of the A.O. such as is visualised on metaphase chromosomes under certain conditions, seems to depend on the DNA/A.O. ratio since a green fluorescence is observable when the A.O. binds to the DNA as a monomer, while a red fluorescence will be obtained if the A.O. binds in an aggregated form. This could explain the homogeneous changes of colour (from green to red) in untreated chromosomes when varying the concentration of the A.O. or the time of staining.

A.O. is a dye capable, in the proper conditions, of discriminating single stranded from double stranded nucleic acids. These criteria have been applied to denatured and reassociated DNA. In this sense, those regions of the chromosome which may arrange the dye in monomers will fluoresce green, while the rest will fluoresce orange. According to previous studies it has been claimed that the double stranded DNA, resulting from the reassociation of hydrogen bonds between complementary purines and pyrimidines in highly repetitive DNA, may arrange the A.O. as monomers. However moderately repetitive or non-repetitive DNA does not have this opportunity since complementary sequences are not re-established. This way of acting of the A.O. has been widely accepted giving a range of colours from green to red (Stockert and Lisanti, 1972; Campbell and Gledhill, 1973).

With this in mind, we have prompted an investigation firstly to analyse the $\mathrm{C}$-positive heterochromatin included in the chromosome complement of the grasshopper Arcyptera fusca, in order to ascertain whether all the C-positive bands show identical staining characteristics after the employment of some treatments on fixed chromosomes and, secondly, to discuss the homogeneity and the heterogeneity of such regions and the characteristics of the A.O. staining of the chromatin. 


\section{MATERIALS AND METHODS}

Males and females of Arcyptera fusca (Orthoptera: Acrididae) collected in the field were used in the present study. Testes of males were fixed in a mixture of glacial acetíc acid and ethanol $(3: 1)$ while females were abdominally injected with 0.05 per cent of colchicine in insect saline solution for $8 \mathrm{~h}$ before the ovarioles and gastric caeca were removed.

The C-banding technique is basically that reported in a previous paper (López-Fernández and Gosálvez, 1981). C-banded slides were stained with $1.6 \times 10^{-7} \mathrm{~g} \mathrm{ml}^{-1}$ acridine orange in phosphate buffer $(p \mathrm{H} \mathrm{6.8)}$ for $5 \mathrm{~min}$, washed and mounted in the same buffer. Once the preparations were observed and photographed they were immersed in absolute methanol for $2 \mathrm{~h}$, rinsed in distilled water and air dried. Some of these slides were then stained with 2 per cent Giemsa in phosphate buffer, rinsed in distilled water, air dried, mounted and photographed.

For denaturation and staining with A.O. oneweek-old preparations were immersed for $3 \mathrm{~min}$ in $2 \times \mathrm{SSC}$ at $96^{\circ} \mathrm{C}$ and then quickly transferred to an A.O. solution $\left(1.6 \times 10^{-7} \mathrm{~g} \mathrm{ml}^{-1}\right)$ phosphate buffer ( $p \mathrm{H} \mathrm{6.8)}$ for $5 \mathrm{~min}$, washed in the same buffer, mounted and observed.

For observations of fluorescence, a Zeiss microscope equipped with an epiluminator and neofluar objectives was used. The cells were photographed by using Valca F22 Film.

\section{RESULTS}

\section{C-banding}

A. fusca presents 23 acrocentric chromosomes in males and 24 elements in females; thus the sex chromosome determinism is of the type $\mathrm{X} 0 / \mathrm{XX}$.

$\mathrm{C}$-banded preparations (somatic and gonial) show dark staining in the centromeric regions of every chromosome and in the telomeres of several autosomes $\left(\mathrm{M}_{3}-\mathrm{S}_{11}\right)$. The heterochromatin located in the telomeres appears as small segments which commonly give rise to polymorphic systems among the populations (fig. 1). Moreover the metaphases from gonial cells (germ line) show an additional large C-band which is absent from both ovariole wall cells and gastric caeca (somatic line). This band is located close to the centromeric one of the $\mathrm{M}_{3}$ pair, and flanks the region where an active NOR is present (Rufas et al., 1983) (compare fig. 2(a) and 2(b)).

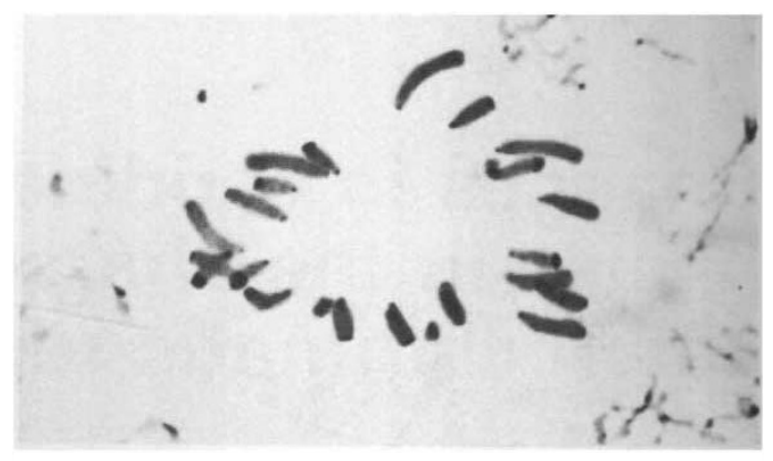

Figure 1 C-banded somatic metaphase from ovariole walls of A. fusca. Note that every chromosome presents a small procentric band, and that some autosomes show telomeric blocks.

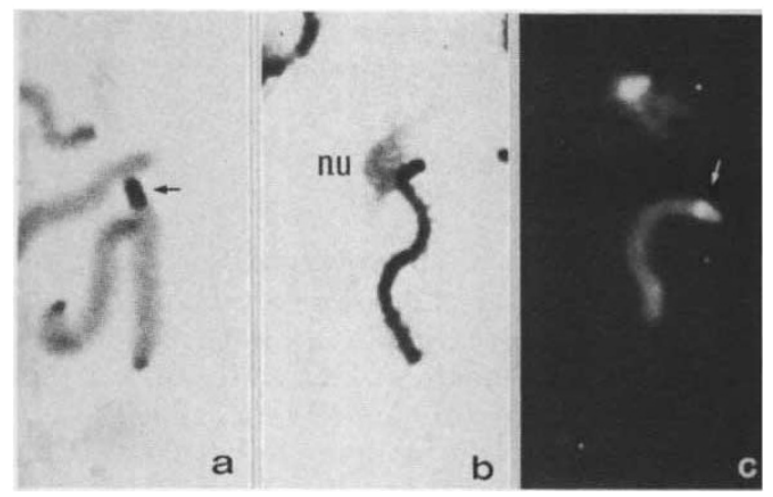

Figure 2 Selected $\mathrm{M}_{3}$ chromosomes from gonial metaphases showing the interstitial band flanking an active NOR. a, $\mathrm{C}$-banding. The interstitial band is located close to the centromeric one. b, Ag-staining. A nucleolus ( $\mathrm{Nu}$ ) appears attached to the region where the interstitial $\mathrm{C}$-band is located. c, A.O. staining after thermal denaturation. The $\mathrm{M}_{3}$ chromosome shows a bright interstitial band.

\section{C-banding plus acridine orange}

When untreated metaphases are stained with a $1.6 \times 10^{-9} \mathrm{~g} \mathrm{ml}^{-1}$ A.O. solution for $3 \mathrm{~min}$, the whole chromosomes fluoresce green or yellow-green while they are red when either the concentration of A.O. is increased to $1.6 \times 10^{-7} \mathrm{~g} \mathrm{ml}^{-1}$ or are stained for time longer than $3 \mathrm{~min}$ with the former concentration. On the contrary, C-banded chromosomes stained with A.O. show certain regions clearly differentiated. The centromeres and the telomeres which are C-positive show green fluorescence while the remaining chromatin fluoresces orange. In every case the $\mathrm{M}_{3}$ pair showed a green fluorescent large block proximally located. Thus, 
the interstitial $\mathrm{C}$-band which characterizes germ line mitotic metaphases and is absent from somatic metaphases is also green in the latter case when stained with A.O. (fig. 3).
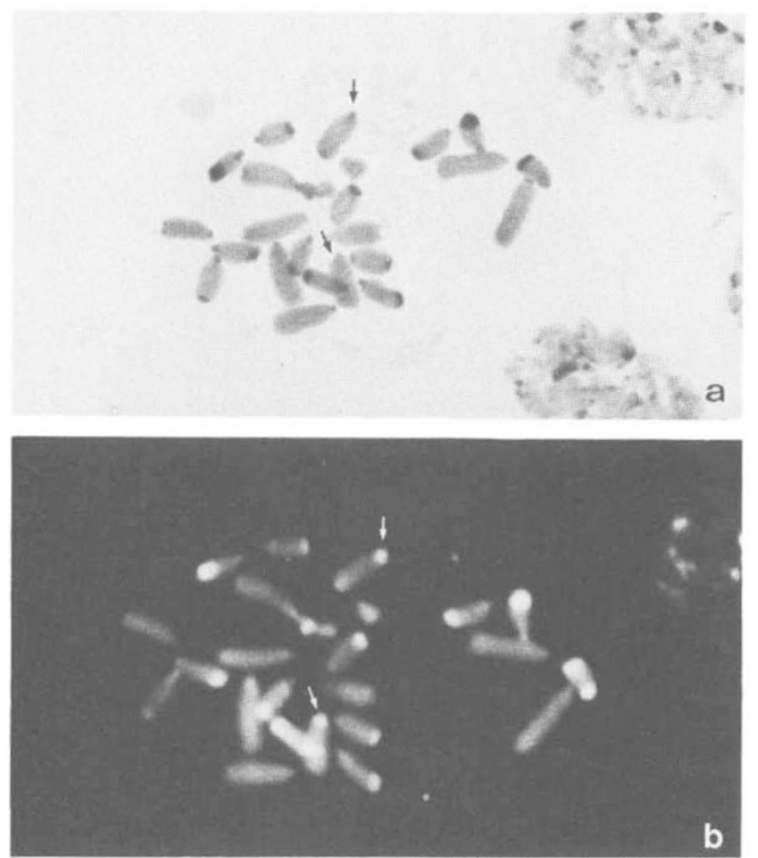

Figure 3 a, C-banded somatic metaphase. Each chromosome presents centromeric bands and several autosomes show distal positive blocks. Note that the $\mathbf{M}_{3}$ chromosomes (arrows) are devoid of any interstitial band. b, Same plate destained and stained with A.O. Identical bands as above are observable, excepting the big fluorescent band located near the centromere of the $\mathbf{M}_{3}$ chromosomes (arrows).

\section{Denaturation and fast reassociation}

The fluorescence pattern of mitotic chromosomes stained with A.O. after thermal "in situ" denaturation differs from that of $\mathrm{C}$-banding. Chromosomes at prophase and metaphase show bright green fluorescent blocks in their centromeric regions. This zone is also green stained in a Bchromosome found in a female (fig. 4(b)). The faintly blue stained chromatin of C-banded metaphases stained with Giemsa fluoresces in orange and the distal heterochromatic segments which show dark blue staining in C-banded chromosomes reveal weak green fluorescence. Unfortunately it is almost impossible to capture this clear differentiation in black and white film given the orange and light green colours. (fig. 4(a, b)) .
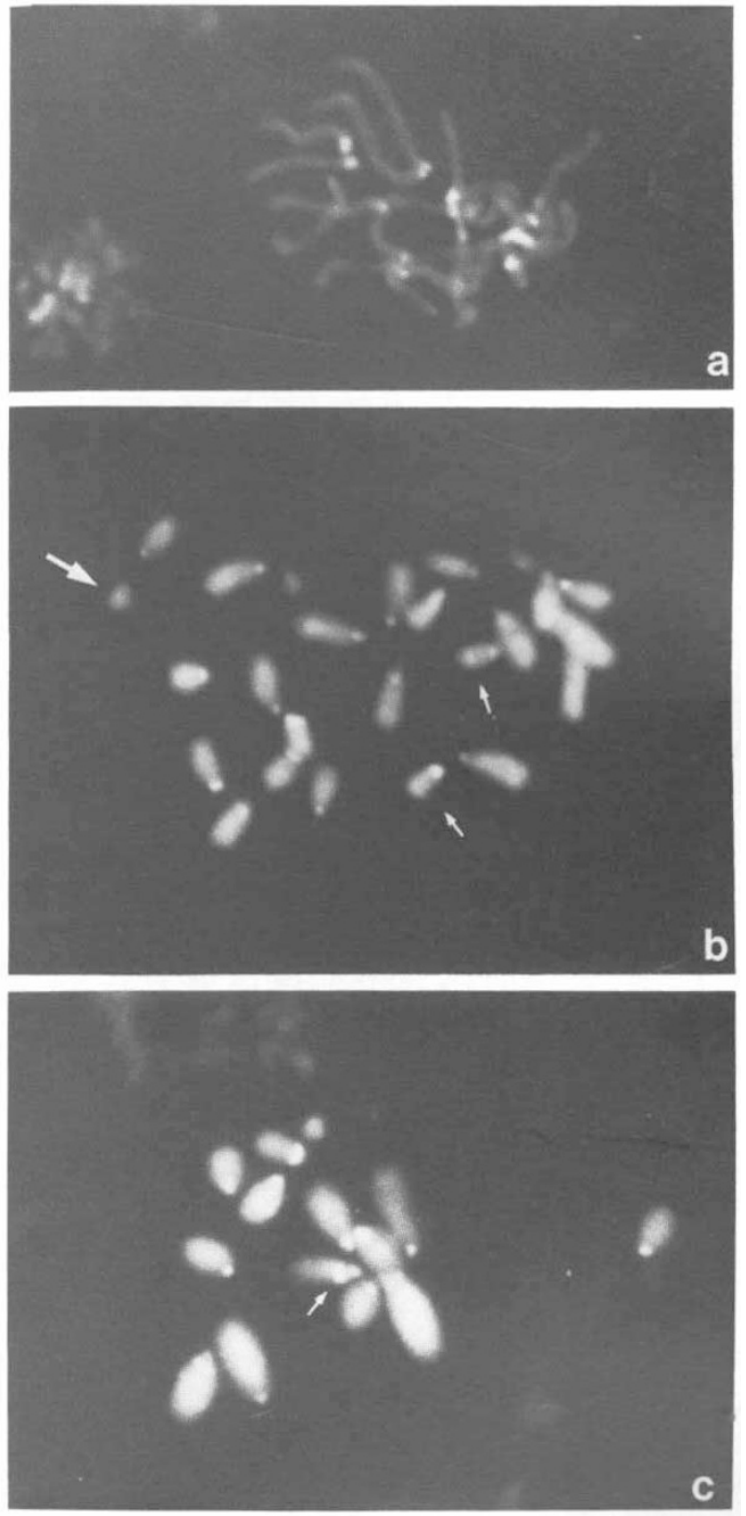

Figure 4 Denatured and reassociated chromosomes from gastric caeca stained with A.O. a, Prophase. Brights bands are visible where the centromeres are located. b, Metaphase. A B-chromosome (big arrow) presents likewise any other chromosome a centromeric band. The distal blocks which characterize several autosomes are detected with difficulty because they fluoresce in weak green (arrows). c, Selected chromosomes. An interstitial thin band is clearly observable in the $\mathbf{M}_{3}$ chromosome.

Besides these bands, the $M_{3}$ pair presents a bright green thin interstitial band near its centromere. This band corresponds to that found in C-banded preparations and is located close to the nucleolar organiser (fig. 2(c) and 4(c)). 


\section{DISCUSSION}

As used in the present case, the way of binding to the chromatin of the A.O. offers the possibility of uncovering the different nature of the heterochromatic regions present in a genome. In the case of A. fusca, C-banding does not discriminate between centromeric and telomeric heterochromatin since both show the same characteristics of staining. However, the discordance in the emission of fluorescence by both types of heterochromatin after thermal denaturation and A.O. staining indicates a clear difference between them. Moreover, some chromosome regions, not detectable after C-banding, are clearly visualised when A.O. staining is used on C-banded slides. This, in turn, indicates a different nature of these particular regions.

The differences in the pattern of green and orange fluorescence obtained after C-banding and heat treatment permit us to assume that not only the characteristics of the DNA (i.e. repetitious or non-repetitious) but also the proteins which are not removed are involved in the binding of A.O. Thus, if the structure of the centromeric chromatin and that surrounding a NOR in $\boldsymbol{A}$. fusca presents a different packing with respect to that of the telomeres they could reassociate differentially under identical conditions of denaturation and reassociation.

In this sense, Burkholder and Weaver (1977) have discussed the role of proteins to explain differences in the staining of chromosomes, and Hagele (1979) suggested the implication of histones to explain the different patterns of A.O. staining observed between positively and negatively $\mathrm{X}$ chromosomes during meiosis after thermal denaturation and reassociation. In the same way Campbell and Gledhill (1973) interpreted certain differences in microfluorometry of individual nuclei from chick embryo stained with A.O. according to the sensitivity of nuclear proteins to different treatments and they suggested a progressive extraction of nuclear proteins by increasing the salt concentration.

John and Miklos (1979) have claimed that heterochromatic regions present a certain rate of heterogeneity. Thus, when some differential stainings have been applied certain heterochromatic regions do not C-band (García de la Vega et al., 1982; Camacho et al., 1985) and in other cases show a great variability in the base richness (Schweizer et al., 1983). This also seems to be case of $A$. fusca. In this species centromeric and telomeric C-bands show differences in A.O. stain- ing which in our opinion may correspond to differences in the chromatin structure, particularly in the protein packing.

Moreover, the interstitial band related to the nucleolar region of the $\mathbf{M}_{3}$ pair behaves in different ways under different conditions. In somatic tissues it is not visible with $\mathrm{C}$-banding, and differs in size when stained with A.O. after denaturation and renaturation. Thus, the procedures employed in the present experiment are very useful to show cryptic differences existing in otherwise homogeneously C-banded regions.

Acknowledgements We would like to thank Dr R. Mezzanotte for his very helpful comments on the manuscript. This work has been supported by C.A.I.C.Y.T. (Spain).

\section{REFERENCES}

BURKHOLDER, G. D. AND WEAVER, M. G. 1977. DNA-protein interaction and chromosome bands. Exp. Cell. Res., 110, 251-262.

CAMACHO, J. P. M., VISERAS, E., NAVAS, J. AND CABRERO, J. 1984. C-heterochromatic content of supernumerary chromosome segments of grasshoppers: detection of an euchromatic extra segment. Heredity, 53, 167-175.

CAMPBELL, G. C. AND GLEDHILL, B. L. 1973. Chromatin of primitive erythroid cells from the chick embryos. I. Changes in Acridine Orange binding and the sensitivity of the thermal denaturation during maturation. Chromosoma, $41,385-394$.

CASSPERSON, T., ZECH, L., MODERT, E. J., FOLEY, G. E., WAGH, V. AND SIMONSSON, E. 1969. DNA binding fluorochromes for the study of metaphase nucleus. Exp. Cell. Res., 58, 141-152.

GARCIA DE LA VEGA, C., LÓPEZ-FERNÁNDEZ, C., GOSÁlVEZ, J. AND RUFAS, J. S. 1982. Cytogenetic studies on Chorthip pus jucundus (Fish) (Orthoptera). II. Heterochromatin variation and NOR localization. Cytobios, 37, 7-14.

HAGELE, K. 1979. Selective staining of $X$ chromosome segments in Schistocerca gregaria after denaturation and reassociation procedures. Chromosoma, 7, 217-225.

JOHN, B., KING, M., SCHWEIZER, D. AND MENDELAK, M. 1985. Equilocality of heterochromatin distribution and heterochromatin heterogeneity in acridoid grasshoppers. Chromosoma, 91, 185-200.

JOHN, B. AND MIKLOS, G. C. L. 1979. Functional aspects of satellite DNA and heterochromatin. Int. Rev. of Cytology, $58,1-100$.

LÓPEZ-FERNÁNDEZ, C. AND GOSÁlVEZ, J. 1981. Differential staining of a heterochromatic zone in Arciptera fusca (Orthoptera). Experientia, 37, 240.

RUFAS, J. S., GOSÁlVEZ, J., LÓPEZ-FERNÁNDEZ, C. AND CARDoso, H. 1983. Complete dependence between Ag NORs and C-positive heterochromatin revealed by simultaneous Ag-NOR C-banding method. Cell. Biol. Int. Rep., 7, 275-281

SAKODA, M., HIROMI, K. AND AKASAKA, K. 1971. Kinetic studies of interaction between acridine orange DNA. Biopolymers, 10, 1003-1012. 
SATO, S. 1980. Differential staining of C-bands with A.O. and Hoechst 33258 in chromosome of Nothoscordum fragans. Cytologia, 45, 127-136.

SCHNEDL, W. R., ABRAHAM, R., FORSTER, M. AND SCHWEIZER, D. 1981. Differential fluorescent staining of porcine heterochromatin by chromomycin A3/distamycin A/DAPI and 287/170. Cytogenet. Cell. Genet, 31, 249-253.

STOCKERT, J. C. AND LISANTI, J. A. 1972. Acridine orange differential.fluorescence of fast and slow-reassociating chromosomal DNA after "in situ" DNA denaturation and reassociation. Chromosoma, 37, 117-130.
SCHWEIZER, D. 1976. Fluorescent chromosome banding in plants: applications, mechanisms and implications for chromosome structure. Proc. 4th. John Innes Symp. 61-72.

SCHWEIZER, D. 1981. Review article: counterstain-enhanced chromosome banding. Hum. Genet., 57, 1-14.

SCHWEIZER, D., MENDELAK, M., WHITE, M. J. D. AND CONTRERAS, N. 1983. Cytogenetics of the parthenogenetic grasshopper Warramaba virgo and its bisexual relatives X. Pattern of fluorescent banding. Chromosoma, 88, 227236. 